CARNETS DE Carnets de géographes

GÉOGRAPHES

$1 \mid 2010$

Varia

\title{
Journées de terrain à Lalibela (Éthiopie)
}

\section{Marie Bridonneau}

\section{CpenEdition}

Journals

Édition électronique

URL : http://journals.openedition.org/cdg/1906

DOI : 10.4000/cdg. 1906

ISSN : 2107-7266

Éditeur

UMR 245 - CESSMA

Référence électronique

Marie Bridonneau, « Journées de terrain à Lalibela (Éthiopie) », Carnets de géographes [En ligne],

1 | 2010, mis en ligne le 01 octobre 2010, consulté le 07 mai 2019. URL : http:// journals.openedition.org/cdg/1906; DOI : 10.4000/cdg.1906

\section{c) (i) $९$}

La revue Carnets de géographes est mise à disposition selon les termes de la Licence Creative Commons Attribution - Pas d'Utilisation Commerciale - Pas de Modification 4.0 International. 


\title{
Journées de terrain à Lalibela (Éthiopie)
}

\author{
Marie Bridonneau \\ Doctorante en géographie, Université Paris Ouest Nanterre la Défense (laboratoire Gecko) \\ ATER à l'université de Provence
}

Près d'un an après mon premier séjour dans les villes du nord de l'Éthiopie, je décidai de me concentrer sur Lalibela et d'y construire, dans le cadre de ma thèse, mon principal terrain d'études. En quelques mois, cette petite ville touristique d'environ 15000 habitants a changé de visage sous l'effet de politiques publiques toujours plus ambitieuses et radicales : des milliers d'habitants ont été contraints de quitter les quartiers centraux et de s'installer dans les nouvelles périphéries - c'est-à-dire le long de la route qui relie Lalibela à son aéroport et aux villes de l'est; les hôtels en construction colonisent les sites surplombant les vallées et offrent des vues susceptibles de séduire l'œil des touristes ; une station essence a vu le jour; le lycée s'agrandit; des arbres sont plantés. Lalibela apparaissait ainsi comme un observatoire privilégié des recompositions spatiales en cours dans les villes éthiopiennes, révélatrices des ambitions économiques et politiques d'un État en quête de modernité et de reconnaissance internationale. Mon ambition de départ était l'analyse des enjeux et impacts des processus de patrimonialisation dans les villes secondaires d'Éthiopie. Première destination touristique du pays (environ 22000 visiteurs par an), Lalibela semblait s'imposer comme un terrain potentiel pour mener ces recherches : la ville renferme en effet onze églises creusées dans la roche qui ont été inscrites sur la Liste du Patrimoine mondial de l'Unesco en 1978.

Suscitant la curiosité d'archéologues comme d'historiens, manne économique pour l'Église orthodoxe éthiopienne qui en taxe l'entrée, lieux de culte et de pèlerinage pour les orthodoxes du pays, marqueurs de l'imagerie nationale, les églises de Lalibela ont été érigées en patrimoine et recouvrent une multitude de valeurs. Je choisissais donc d'y interroger ces valeurs ainsi que les représentations et pratiques qui leur sont associées.

Aujourd'hui, mon questionnement a évolué d'une approche très sectorielle - par le patrimoine - vers une approche plus territoriale et multiscalaire. De nouvelles réflexions se sont nouées au cours de mes allersretours entre Lalibela, Addis Abeba et Nanterre. À l'origine de ces cheminements réside en partie ma pratique quotidienne de la ville, durant laquelle les temps d'observation sont concomitants à ceux des entretiens. C'est dans ces moments que j'ai pu percevoir la force de l'altérité existante entre les habitants et les représentants d'un système mondialisé, qu'il s'agisse des touristes ou des normes internationales parcourant Lalibela. Cette altérité exigeait alors d'être déclinée selon différentes temporalités qui organisent le partage du bien patrimonialisé, mais aussi de l'espace tout entier de Lalibela.

Je suis une fille, jeune, je suis blanche, à pieds et seule : c'est à peu près comme cela que j'étais vue et décrite par les habitants qui me voyaient déambuler dans Lalibela du matin au soir. Avant d'être reconnue 
comme une apprentie chercheuse, j'ai été perçue comme quelqu'un venu d'ailleurs, au même titre que les touristes s'arrêtant une ou deux journées afin de visiter les églises. La catégorisation me semblait très forte entre Farendj ${ }^{1}$ et Abasha $^{2}$ et, avant d'entreprendre des entretiens destinés à appréhender les pratiques et représentations associées aux politiques patrimoniales, j'ai décidé d'interroger les rapports qui s'établissent entre ceux qui se considèrent d'ici - qu'ils viennent des campagnes environnantes ou qu'ils soient tout juste de retour à Lalibela après de longues décennies passées en Europe - et jaugent ceux venus d'ailleurs, ceux-là venus jusqu'en Éthiopie pour vivre un peu d'aventure. Marcher dans Lalibela et voir se répéter les mêmes scènes du petit garçon qui court après un groupe de touristes pour leur vendre quelques souvenirs, du visiteur qui se plaint de toujours payer «plus cher», des prêtres qui traquent celui qui ne se serait pas acquitté de l'achat d'un ticket pour accéder aux églises, du voyageur qui distribue des stylos aux écolier, etc. m'a permis d'identifier un des enjeux de l'arrimage de Lalibela au système touristique mondialisé : l'argent. En remontant chaque jour la rue principale qui dessert les églises, en me rendant au café internet, en allant prendre un thé et en écoutant les rumeurs de la ville, j'ai réalisé que pour de nombreuses personnes, jeunes en particulier, le visiteur de passage est un sponsor potentiel. Beaucoup d'entre eux ont par exemple en mémoire l'histoire du Mountain View Hotel : de standard international, offrant une vue imprenable sur les montagnes environnant Lalibela, il est la propriété de deux anciens guides locaux. La légende raconte que l'un deux, ayant accueilli un touriste fortuné à sa descente de l'avion, l'emmena au bord de l'escarpement et lui raconta son rêve d'y construire, un jour, un hôtel. Quelques semaines plus tard, plusieurs millions de birr $^{3}$ étaient versés sur son compte en banque. À quelques dizaines de mètres de là, un autre hôtel est aujourd'hui en construction : son propriétaire est surnommé ironiquement «Plan International», en référence à une Organisation Non Gouvernementale omniprésente à Lalibela qui finance des projets de développement (construction de jardins d'enfants, amélioration de l'accès à l'eau, etc.) en demandant aux citoyens des pays riches de parrainer un enfant d'un pays pauvre. Ce jeune homme aurait ainsi obtenu le «parrainage » d'une multitude de touristes rencontrés lors de leur passage à Lalibela et ainsi rassemblé assez de fonds pour entreprendre la construction de cet hôtel. Un jeune étudiant de la Preparatory School ${ }^{4}$ de Lalibela, âgé de 19 ans, raconte fièrement les stratégies qu'il élabore face aux touristes afin de s'enrichir : «j'ai mis en place une véritable stratégie. D'abord, j'aborde uniquement les touristes âgés car ce sont eux qui sont les plus riches. Je leur montre mes résultats scolaires qui sont très bons. Je leur présente ma mère, qui les invite chez nous pour la cérémonie du café. Je leur raconte à quel point nous sommes pauvres. Sans rien leur demander, je les emmène dans la montagne, jusqu'à l'église d'Asheton ${ }^{5}$. S'ils me proposent de l'argent, je refuse. Ils me considèrent alors comme un bon garçon et estiment que je mérite leur soutien. Ainsi chaque mois, je reçois plusieurs milliers de birr par Western Union, provenant de différents touristes. Mais ce n'est pas assez. ». Dans les cafés internet, on peut alors rencontrer de jeunes garçons qui envoient des mails de courtoisie à ceux qu'ils appellent « père » et «mère » mais aussi des courriers de rappels quand l'envoi régulier d'argent a brutalement cessé. Il semble ainsi qu'aujourd'hui les touristes soient de plus en plus identifiés comme une source de revenus légitimes, presque de droit, pour de nombreux jeunes gens de Lalibela.

Dans la perspective de comprendre le rôle des éléments patrimonialisés dans l'organisation socio-spatiale de Lalibela, je me trouvais, suite à ces premiers moments de terrain, assez déroutée : la valeur économique des églises semblait prendre le pas sur toute autre valeur, religieuse ou identitaire. De prime abord, les gens que

\footnotetext{
${ }^{1}$ Le terme farendj est utilisé en Ethiopie pour désigner l'étranger mais aussi le « blanc ».

${ }^{2}$ Le terme Abasha est utilisé par les Ethiopiens des hauts plateaux pour se désigner.

${ }^{3}$ Le birr est la monnaie éthiopienne. A titre indicatif, le salaire d'un enseignant en lycée est d'environ 1000 birr en début de carrière, celui d'une serveuse est compris entre 80 birr et 150 birr par mois.

${ }^{4}$ La Preparatory School, comme son nom l'indique, « prépare » les jeunes étudiants ayant achevé leur scolarité primaire et secondaire à accéder à l'enseignement supérieur. Cette formation très générale s'étend sur deux ans.

${ }^{5}$ Asheton est à la fois le nom d'une montagne surplombant Lalibela, mais aussi celui d'un petit village et d'une église creusée dans la montagne. C'est l'objet d'une excursion pour de nombreux touristes qui restent plus d'une journée à Lalibela.
} 
je rencontrais me parlaient bien plus du tourisme et des touristes que des églises. Dans quelle mesure, frappée par les transformations socio-spatiales liées à cette activité, n'avais-je pas intuitivement concentré mon regard sur cet objet et mis de côté les monuments qui m'apparaissaient alors seulement comme un instrument mis au service des politiques nationales de développement économique ? Que devenait alors mon approche par les enjeux et impacts des processus patrimoniaux à Lalibela ?

C'est de nouveau par mes parcours dans la ville et par les rencontres occasionnées que j'ai essayé de dépasser l'identification du seul enjeu économique dans la construction de mon objet de recherches. Je me suis notamment intéressée à d'autres temporalités : plus tôt le matin, le dimanche, les jours célébrant des saints particuliers ${ }^{6}$, etc. Avant le lever du soleil, de nombreuses personnes vêtues de blanc, plutôt des femmes et des personnes âgées, se rendent à la messe et prient des heures durant à l'intérieur et autour des églises. Vers sept heures, la plupart des véhicules de tourisme quittent les cours des hôtels nichés dans les zones touristiques pour rejoindre à leur tour les églises. À partir de huit heures, des groupes d'étudiants à pieds et en uniformes remplissent les rues et rejoignent les différents établissements scolaires de Lalibela alors que les touristes désertent les espaces publics -exceptions faites des trajets qu'ils réalisent pour se rendre d'un groupe d'églises à l'autre - pour ne réapparaître aux abords des magasins de souvenirs qu'entre dix-sept et dix-neuf heures. Pendant ce temps, les femmes affluent des campagnes pour vendre leurs fagots de bois aux restaurants qui les utiliseront comme combustibles dans les cuisines, les employés quittent les bureaux le temps de leur pause-déjeuner, les petits cireurs de chaussures attendent le client sur le bord de la route, etc. Ces balancements dans l'occupation des espaces publics n'a pas cours le dimanche : ce jour-là, la messe rassemble bien plus de croyants, jusque tard dans la matinée. Les touristes, indifférents à ce rythme hebdomadaire et accompagnés des passeurs qui leur donnent accès à Lalibela guides locaux, chauffeurs de 4X4 et de minibus, etc. - poursuivent leurs parcours entre les églises.

$\mathrm{Au}$ moment de Noël, ce sont plus de 100000 pèlerins qui se rendent à Lalibela : les églises sont alors investies par les fidèles et incarnent au mieux la dimension sacrée d'un patrimoine religieux approprié par les croyants ${ }^{7}$. Les touristes étrangers sont aussi présents : ils viennent admirer non seulement les églises mais aussi la dimension «immatérielle ${ }^{8} »$ de ce patrimoine mondial. Chaque temps révèle une nouvelle facette de Lalibela, dans laquelle on peut lire la place prise par les églises, comme un potentiel économique mais aussi en tant que marqueur des identités locales ou point d'ancrage de l'identité religieuse. Aborder la multiplicité des temporalités m'a ainsi permis d'appréhender la pluralité du territoire étudié et de son principal lieu symbolique, les églises.

Finalement, la forte altérité que j'identifiais et ressentais au cours des premières journées passées à Lalibela - entre les habitants permanents et ceux qui n'étaient que de passage - constitua le point de départ d'une réflexion sur la mise en relation du territoire de Lalibela au système mondialisé. Cette connexion brutale et tardive est soutenue par l'implantation de projets de développement financés par la Banque mondiale ou l'Union Européenne, permettant ainsi la circulation des normes internationales. La relation de Lalibela au Monde est également investie par l'État éthiopien qui organise et contrôle le développement économique et urbain par des politiques volontaristes, comme l'illustre le projet en cours de resettlement consistant à vider les abords des églises de ses habitants. Ce déplacement forcé est lui-même justifié par des arguments patrimoniaux - pour protéger la valeur sacrée et architecturale des églises -, mais aussi économiques - afin de rendre le site plus attractif pour les touristes. S'articule dans la petite ville du Lasta ${ }^{9}$, un triangle d'échelles d'action - locale, nationale et internationale - dont les stratégies et les rôles se construisent, se

\footnotetext{
${ }^{6}$ Dans l'Ethiopie orthodoxe, à l'occasion des jours de célébration d'un saint, l'église portant le nom de ce dernier deviendra lieu de prières et festivités particulières.

${ }^{7}$ Il est ici fait référence au noël orthodoxe, célébré début janvier.

${ }^{8}$ Selon la terminologie utilisée par l'Unesco.

${ }^{9}$ Région historique à laquelle appartient Lalibela
} 
rencontrent et s'opposent, organisant l'intégration de Lalibela au sein d'un territoire patrimonial et touristique mondial.

À ces longues heures d'observation et d'interaction inattendue, j'ai toujours associé des journées d'entretiens guidés qui me permettaient d'identifier les discours et représentations sur les églises, le tourisme, la ville et le changement. L'association dialectique de ces deux dimensions du travail de terrain a nourri mon objet de recherches. En outre, les fréquents séjours à Addis Abeba m'ont conduite à davantage intégrer les enjeux nationaux et internationaux à l'origine des recompositions spatiales évoquées. À Lalibela, les paysages se transforment tout comme les rapports sociaux : les maisons sont détruites quand les hôtels se construisent, les vieilles dames embrassent les églises dont elles sont tenues à l'écart quand un petit garçon apprend à amadouer le touriste qui le photographie, etc. Sur ce territoire, on voit la petite ville libérale émerger sous le contrôle paradoxal de la puissance publique. 